PROCEEDINGS OF THE

AMERICAN MATHEMATICAL SOCIETY

Volume 132, Number 9, Pages 2723-2732

S 0002-9939(04)07496-9

Article electronically published on April 8, 2004

\title{
COMPUTING INFIMA ON CONVEX SETS, WITH APPLICATIONS IN HILBERT SPACES
}

\author{
DOUGLAS BRIDGES, HAJIME ISHIHARA, AND LUMINIŢA VÎŢĂ
}

(Communicated by Jonathan M. Borwein)

\begin{abstract}
Using intuitionistic logic, we prove that under certain reasonable conditions, the infimum of a real-valued convex function on a convex set exists. This result is then applied to problems of simultaneous approximation in Hilbert space $H$ and the corresponding operator space $\mathcal{B}(H)$. This enables us to establish that a bounded, weak-operator totally bounded, convex subset of $\mathcal{B}(H)$ is strong-operator located.
\end{abstract}

\section{INTRODUCTION}

In constructive mathematics - mathematics using intuitionistic, rather than classical, logid - the existence of infima of nonempty subsets of $\mathbf{R}$ that are bounded below is not guaranteed. Indeed, the constructive greatest-lower-bound principle states that a nonempty subset $S$ of $\mathbf{R}$ that is bounded below has an infimum if and only if the following condition holds:

(*) for all real numbers $\alpha, \beta$ with $\alpha<\beta$, either $s>\alpha$ for all $s \in S$ or else there exists $s \in S$ with $s<\beta$ (cf. [2], page 37).

A simple application of this principle then shows that $S$ has an infimum if and only if for each $\varepsilon>0$ there exists $s_{0} \in S$ such that $s_{0} \leq s+\varepsilon$ for all $s \in S$.

The additional requirement $(*)$ for the existence of the infimum of $S$ poses problems in many aspects of constructive analysis. For example, given an arbitrary point $x$ in a metric space $(X, \rho)$ and an arbitrary nonempty subset $S$ of $X$, we cannot hope to compute the distance

$$
\rho(x, S)=\inf \{\rho(x, s): s \in S\}
$$

from $x$ to $S$. If we can compute that distance for all $\in X$, then we say that the set $S$ is located.

Received by the editors January 6, 2003.

2000 Mathematics Subject Classification. Primary 03F60, 46 S30.

Key words and phrases. Functional analysis, constructive mathematics.

Bridges and Viţă gratefully acknowledge the support of the Marsden Fund and FoRST New Zealand. All three authors thank the Japan Advanced Institute of Science \& Technology for supporting the visit by the first two during which much of this work was carried out.

${ }^{1}$ Background material in constructive mathematics (of which very little is needed to cope with this paper) can be found in [1, 2, 5, 10]. 
Likewise, given a bounded linear mapping $T: X \rightarrow Y$ between normed spaces, we cannot guarantee that its norm,

$$
\|T\|=\sup \{\|T x\| ; x \in X,\|x\| \leq 1\},
$$

exists; if the norm does exist, then we say that $T$ is normable. It is worth notingalthough we do not use this fact - that a nonzero bounded linear functional on a normed space is normable if and only if its kernel is located.

There are three main results in the present paper. The first, Theorem 2.2 provides conditions under which a convex function that is bounded below on a convex set in a normed space has an infimum. The next, Theorem 3.4 applies this to generalize Ishihara's result ([8], Theorem 4) about the locatedness of convex subsets of a Hilbert space $H$. Our final theorem, Theorem 3.8, uses Theorem 2.2 to prove that a bounded, weak-operator totally bounded, convex subset of $\mathcal{B}(H)$ is strong-operator located, a result that may prove valuable in the constructive theory of operator algebras (cf. [6, 4]).

\section{INFIMA OF CONVEX FUNCTIONS}

Let $X$ be a normed space, and let $X^{*}$ be the space of all bounded (but not necessarily normable) linear functionals on $X$. A subset $S$ of $X$ is said to be weakly totally bounded if for each normable $x^{*} \in X^{*}$ the set $\left\{\left\langle x, x^{*}\right\rangle: x \in S\right\}$ is totally bounded in $\mathbf{C}$. Every ball in $X$ is weakly totally bounded. It is shown in [7] (Proposition 3) that a convex subset $C$ of $\mathbf{C}^{n}$ is totally bounded if and only if the supremum

$$
\sup \{\operatorname{Re} f(x): x \in C\}
$$

exists for each linear functional $f$ on $\mathbf{C}^{n}$, from which it follows that a subset $S$ of a normed space $X$ is weakly totally bounded if and only if the supremum

$$
\sup \left\{\operatorname{Re}\left\langle x, x^{*}\right\rangle: x \in S\right\}
$$

exists for each normable linear functional $x^{*}$ on $X$.

Let $C$ be a nonempty subset of $X$, and let $f$ be a mapping of $C$ into $\mathbf{R}$. We say that $f$ is uniformly differentiable on $C$ if there exists a mapping $x \rightsquigarrow x^{*}$, taking $C$ into the set of normable linear functionals on $X$, such that for each $\varepsilon>0$ there exists $\delta>0$ such that

$$
\left|f(x)-f(u)-\left\langle x-u, x^{*}\right\rangle\right| \leq \varepsilon\|x-u\|
$$

whenever $x, u \in C$ and $\|x-u\|<\delta$. The mapping $x \rightsquigarrow x^{*}$ of $C$ into $X^{*}$ is then called the derivative of $f$ (on $C$ ).

A mapping $f$ of a convex subset $C$ of $X$ into $\mathbf{R}$ is said to be convex if for all $x, y \in C$ and $\lambda \in[0,1]$,

$$
f(\lambda x+(1-\lambda y)) \leq \lambda f(x)+(1-\lambda) f(y) .
$$

Lemma 2.1. Let $C$ be a bounded convex subset of a normed space $X$ such that $\sup \left\{\left\langle y, x^{*}\right\rangle: y \in C\right\}$ exists for each $x^{*} \in X^{*}$. Let $f$ be a uniformly differentiable convex mapping of $C$ into $\mathbf{R}$. Then for each $\varepsilon>0$ there exists $\tau>0$ such that if $x \in C$, then

- either $f(x) \leq f(y)+\varepsilon$ for all $y \in C$,

- or there exists $z \in C$ such that $f(z)<f(x)-\tau \varepsilon$. 
Proof. Choose $M>1$ such that $\|x-y\| \leq M$ for all $x, y \in C$. Let $\varepsilon>0$, and choose $\delta \in(0,2 M)$ such that for each $x \in C$ there exists a normable $x^{*} \in X^{*}$ such that

$$
\left|f(x)-f(u)-\left\langle x-u, x^{*}\right\rangle\right| \leq \frac{\varepsilon}{8 M}\|x-u\|
$$

whenever $u \in C$ and $\|x-u\|<\delta$. Fix $x \in C$, and construct the corresponding normable linear functional $x^{*}$. Either $\sup _{u \in C}\left\langle x-u, x^{*}\right\rangle<\varepsilon / 2$ or else $\sup _{u \in C}\left\langle x-u, x^{*}\right\rangle$ $>\varepsilon / 4$. In the first case, given $u \in C$ and setting

$$
\lambda=\frac{\delta}{2 M}, \quad y=(1-\lambda) x+\lambda u,
$$

we have

$$
\|x-y\|=\lambda\|x-u\| \leq \lambda M=\frac{\delta}{2}
$$

Hence

$$
\begin{aligned}
\frac{\varepsilon}{8} \lambda & =\frac{\varepsilon}{8 M} \lambda M \\
& \geq \frac{\varepsilon}{8 M}\|x-y\| \\
& \geq f(x)-f(y)-\left\langle x-y, x^{*}\right\rangle \\
& \geq f(x)-(1-\lambda) f(x)-\lambda f(u)-\lambda\left\langle x-u, x^{*}\right\rangle \\
& =\lambda\left(f(x)-f(u)-\left\langle x-u, x^{*}\right\rangle\right) .
\end{aligned}
$$

Dividing throughout by $\lambda$ and rearranging, we obtain

$$
f(x) \leq f(u)+\frac{\varepsilon}{8}+\left\langle x-u, x^{*}\right\rangle<f(u)+\frac{\varepsilon}{8}+\frac{\varepsilon}{2}<f(u)+\varepsilon .
$$

In the case $\sup _{u \in C}\left\langle x-u, x^{*}\right\rangle>\varepsilon / 4$, choose $u \in C$ with $\left\langle x-u, x^{*}\right\rangle>\varepsilon / 4$ and define $\lambda, y$ as in 2.1. Then

$$
-\frac{\varepsilon}{8} \lambda \leq f(x)-f(y)-\left\langle x-y, x^{*}\right\rangle .
$$

So

$$
\begin{aligned}
f(y) & \leq f(x)+\frac{\varepsilon}{8} \lambda-\lambda\left\langle x-u, x^{*}\right\rangle \\
& <f(x)+\lambda\left(\frac{\varepsilon}{8}-\frac{\varepsilon}{4}\right) \\
& =f(x)-\lambda \frac{\varepsilon}{8} \\
& =f(x)-\tau \varepsilon
\end{aligned}
$$

where $\tau=\delta /(16 M)$.

Theorem 2.2. Let $C$ be a nonempty, bounded, weakly totally bounded, convex subset of a normed space $X$, and $f$ a uniformly differentiable, convex mapping of $C$ into $\mathbf{R}$ that is bounded below. Then $\inf f$ exists.

Proof. Without loss of generality, we may assume that $f(x) \geq 0$ for each $x \in C$. It is enough to find, for each $\varepsilon>0$, a point $x \in C$ such that $f(x) \leq f(y)+\varepsilon$ for all $y \in C$. To this end, fixing $x_{1} \in C$ and using Lemma 2.1, construct a sequence $\left(x_{n}\right)$ in $C$ and an increasing binary sequence $\left(\lambda_{n}\right)$ such that

$\triangleright$ if $\lambda_{n}=0$, then $f\left(x_{n+1}\right)<f\left(x_{n}\right)-\tau \varepsilon$; 
$\triangleright$ if $\lambda_{n}=1-\lambda_{n-1}$, then $f\left(x_{n}\right) \leq f(y)+\varepsilon$ for all $y \in C$, and $x_{k}=x_{n}$ for all $k>n$.

We may assume that $\lambda_{1}=0$. Choose a positive integer $N$ such that $f\left(x_{1}\right)-N \tau \varepsilon<0$. If $\lambda_{N}=0$, then $f\left(x_{N+1}\right)<f\left(x_{1}\right)-N \tau \varepsilon<0$, a contradiction. Hence $\lambda_{N}=1$, and we are through.

\section{Applichtions to approximation problems}

In this section we show how Theorem 2.2 can be applied to solve two approximation problems. We first give a couple of elementary lemmas.

Lemma 3.1. Let $a_{1}, \ldots, a_{n}$ be nonnegative numbers, and let $\delta$ be a positive number. Then

$$
0<\sum_{i=1}^{n}\left(a_{i}^{2}+\delta\right)^{1 / 2}-\sum_{i=1}^{n} a_{i} \leq n \delta^{1 / 2} .
$$

Proof. For each $i$ we have

$$
\begin{aligned}
0 & <\left(a_{i}^{2}+\delta\right)^{1 / 2}-a_{i} \\
& =\frac{\left(\left(a_{i}^{2}+\delta\right)^{1 / 2}-a_{i}\right)\left(\left(a_{i}^{2}+\delta\right)^{1 / 2}+a_{i}\right)}{\left(a_{i}^{2}+\delta\right)^{1 / 2}+a_{i}} \leq \frac{a_{i}^{2}+\delta-a_{i}^{2}}{\delta^{1 / 2}}=\delta^{1 / 2} .
\end{aligned}
$$

Summing over $i$, we obtain the desired inequalities.

Lemma 3.2. Let $f_{1}, \ldots, f_{n}$ be mappings of a set $S$ into $\mathbf{R}^{0+}$ such that for each $\delta>0$ the infimum

$$
\inf \left\{\sum_{i=1}^{n}\left(f_{i}(y)^{2}+\delta\right)^{1 / 2}: y \in S\right\}
$$

exists. Then $\inf \left\{\sum_{i=1}^{n} f_{i}(y): y \in S\right\}$ exists.

Proof. Given $\varepsilon>0$, set $\delta=\left(\frac{\varepsilon}{2 n}\right)^{2}$ and choose $y_{0} \in S$ such that

$$
\sum_{i=1}^{n}\left(f_{i}\left(y_{0}\right)^{2}+\delta\right)^{1 / 2} \leq \sum_{i=1}^{n}\left(f_{i}(y)^{2}+\delta\right)^{1 / 2}+\frac{\varepsilon}{2} \quad(y \in S) .
$$

For each $y \in S$, Lemma 3.1 then shows that

$$
\begin{aligned}
\sum_{i=1}^{n} f_{i}\left(y_{0}\right) & <\sum_{i=1}^{n}\left(f_{i}\left(y_{0}\right)^{2}+\delta\right)^{1 / 2} \\
& \leq \sum_{i=1}^{n}\left(f_{i}(y)^{2}+\delta\right)^{1 / 2}+\frac{\varepsilon}{2} \\
& \leq \sum_{i=1}^{n} f_{i}(y)+n \delta^{1 / 2}+\frac{\varepsilon}{2}=\sum_{i=1}^{n} f_{i}(y)+\varepsilon
\end{aligned}
$$

The desired result now follows from an observation made in the introduction.

Proposition 3.3. The norm $\|\cdot\|$ on a Hilbert space $H$ is uniformly differentiable on any set that is bounded away from 0 . In fact, if $0<\varepsilon<1,\|x\|>r>0$, and $0<\|h\|<r \varepsilon / 2$, then

$$
0 \leq\|x+h\|-\|x\|-\left\langle h, \frac{x}{\|x\|}\right\rangle \leq \varepsilon\|h\| .
$$


Proof. Let $x, h$ be vectors in $H$ with $0<\|h\|<\|x\|$. Then

$$
\begin{aligned}
\|x\|-\|x-h\| & \leq\|x\|-\left\langle x-h, \frac{x}{\|x\|}\right\rangle \\
& =\left\langle x-(x-h), \frac{x}{\|x\|}\right\rangle=\left\langle h, \frac{x}{\|x\|}\right\rangle \\
& =\left\langle x+h-x, \frac{x}{\|x\|}\right\rangle \\
& =\left\langle x+h, \frac{x}{\|x\|}\right\rangle-\|x\| \\
& \leq\|x+h\|-\|x\| .
\end{aligned}
$$

Hence

$$
0 \leq\|x+h\|-\|x\|-\left\langle h, \frac{x}{\|x\|}\right\rangle \leq\|x+h\|-\|x-h\|-2\|x\| .
$$

Set

$$
u=\frac{x+h}{\|x+h\|}, \quad v=\frac{x-h}{\|x-h\|} .
$$

Then the right-hand side of (3.1) becomes

$$
\begin{aligned}
\langle x+h, u\rangle+\langle x-h, v\rangle-2\|x\| & \leq\|x\|+\langle h, u\rangle+\|x\|-\langle h, v\rangle-2\|x\| \\
& =\langle h, u-v\rangle .
\end{aligned}
$$

Hence

$$
0 \leq\|x+h\|-\|x\|-\left\langle h, \frac{x}{\|x\|}\right\rangle \leq\|h\|\|u-v\| .
$$

Now,

So

$$
\begin{aligned}
\frac{x+h}{\|x+h\|}-\frac{x}{\|x\|} & =\left(\frac{x+h}{\|x+h\|}-\frac{x}{\|x+h\|}\right)+\left(\frac{x}{\|x+h\|}-\frac{x}{\|x\|}\right) \\
& =\frac{h}{\|x+h\|}+\frac{\|x\|-\|x+h\|}{\|x+h\|\|x\|} x .
\end{aligned}
$$

Likewise,

$$
\left\|\frac{x+h}{\|x+h\|}-\frac{x}{\|x\|}\right\| \leq \frac{\|h\|}{\|x+h\|}+\frac{|\|x\|-\|x+h\||}{\|x+h\|} \leq \frac{2\|h\|}{\|x+h\|} .
$$

Hence

$$
\left\|\frac{x-h}{\|x-h\|}-\frac{x}{\|x\|}\right\| \leq \frac{2\|h\|}{\|x-h\|}
$$

$$
\begin{aligned}
\|u-v\| & \leq\left\|\frac{x+h}{\|x+h\|}-\frac{x}{\|x\|}\right\|+\left\|\frac{x-h}{\|x-h\|}-\frac{x}{\|x\|}\right\| \\
& \leq 2\|h\|\left(\frac{1}{\|x+h\|}+\frac{1}{\|x-h\|}\right) .
\end{aligned}
$$

If $r>0,0<\varepsilon<1$, and $0<\|h\|<r \varepsilon / 2$, then, by the foregoing and (3.2), we have

$$
0 \leq\|x+h\|-\|x\|-\left\langle h, \frac{x}{\|x\|}\right\rangle \leq \frac{4\|h\|}{r / 2}\|h\| \leq 4 \varepsilon\|h\| .
$$

This completes the proof. 
If $f, g$ are uniformly differentiable mappings of a subset $C$ of a Hilbert space $H$ into $\mathbf{R}$, with derivatives $x \rightsquigarrow x_{f}^{*}$ and $x \rightsquigarrow x_{g}^{*}$ on $C$, then $f+g$ is differentiable, and has derivative $x \rightsquigarrow x_{f}^{*}+x_{g}^{*}$, on $C$. Note that, as an example in [7] shows, it cannot be proved constructively that the sum of two normable linear functionals on a Banach space is normable; hence it also cannot be proved that the sum of two differentiable mappings in that more general context is differentiable.

We now have the first of our two approximation results.

Theorem 3.4. Let $C$ be a bounded, weakly totally bounded, convex subset of a Hilbert space $H$, and let $x_{1}, \ldots, x_{n}$ be points of $H$. Then

$$
\inf \left\{\sum_{i=1}^{n}\left\|x_{i}-y\right\|: y \in C\right\}
$$

exists.

Proof. Assume, to begin with, that $\rho\left(x_{i}, C\right)>0$ for each $i$.2 Then Proposition 3.3 shows that each of the convex functions $y \rightsquigarrow\left\|x_{i}-y\right\|$ is uniformly differentiable on $C$, as therefore is the convex function $y \rightsquigarrow \sum_{i=1}^{n}\left\|x_{i}-y\right\|$. Applying Theorem 2.2 to the latter function, we obtain the desired infimum.

It remains to remove the condition that $\rho\left(x_{i}, C\right)>0$ for each $i$. We accomplish this as follows. Let $H^{\prime}$ be the Hilbert space $H \oplus H$. Picking a unit vector $e$ in $H$, and given any $\delta>0$, define $C^{\prime}=C \oplus\left\{\delta^{1 / 2} e\right\}$ and $x_{i}^{\prime}=\left(x_{i}, 0\right) \quad(1 \leq i \leq n)$. Then $C^{\prime}$ is a bounded, weakly totally bounded, convex subset of $H^{\prime}$. Also, for each $i$ and each $y^{\prime}=\left(y, \delta^{1 / 2} e\right)$ in $C^{\prime}$,

$$
\left\|x_{i}^{\prime}-y^{\prime}\right\|=\sqrt{\left\|x_{i}-y\right\|^{2}+\delta} \geq \delta^{1 / 2}
$$

so $\rho\left(x_{i}^{\prime}, C^{\prime}\right)>0$. By the first part of the proof, the quantity

$$
\begin{aligned}
m_{\delta} & =\inf \left\{\sum_{i=1}^{n}\left\|x_{i}^{\prime}-y^{\prime}\right\|: y^{\prime} \in C^{\prime}\right\} \\
& =\inf \left\{\sum_{i=1}^{n}\left(\left\|x_{i}-y\right\|^{2}+\delta\right)^{1 / 2}: y \in C\right\}
\end{aligned}
$$

exists. The desired conclusion now follows from Lemma 3.2

In the special case $n=1$, we obtain the following result from 8 .

Corollary 3.5. Let $C$ be a bounded, convex subset of a Hilbert space $H$ such that $\sup \left\{\left\langle x, x^{\prime}\right\rangle: x \in C\right\}$ exists for each $x^{\prime} \in X$. Then $C$ is located in $H$.

Our second application of Theorem 2.2 deals with linear subsets of $\mathcal{B}(H)$, where $H$ is a Hilbert space. It requires one more lemma and some constructions.

Lemma 3.6. Let $H$ be a direct sum $\bigoplus_{i=1}^{n} H_{i}$ of finitely many Hilbert spaces, and denote by $\mathrm{pr}_{i}$ the mapping of $H$ onto $H_{i}$ that takes $\mathbf{x}=\left(x_{1}, \ldots, x_{n}\right)$ to $x_{i}$. Then $\left\|\operatorname{pr}_{i}(\cdot)\right\|$ is uniformly differentiable on each subset $S$ of $H$ for which $\operatorname{pr}_{i}(S)$ is bounded away from 0.

\footnotetext{
${ }^{2}$ The inequality $\rho\left(x_{i}, C\right)>0$ is not predicated on the existence of the distance from $x_{i}$ to $C$. Rather, it is a shorthand for the statement that $x_{i}$ is bounded away from $C$.
} 
Proof. Let $S$ be such a subset of $H$, and let $\varepsilon>0$. We see from Proposition 3.3 that there exists $\delta>0$ such that if $x_{i}, u_{i} \in H_{i}$ and $\left\|x_{i}-u_{i}\right\|<\delta$, then

$$
\left|\left\|x_{i}\right\|-\left\|u_{i}\right\|-\left\langle x_{i}-u_{i}, \frac{x_{i}}{\left\|x_{i}\right\|}\right\rangle\right| \leq \varepsilon\left\|x_{i}-u_{i}\right\| .
$$

Let $\mathbf{x}, \mathbf{u} \in H$ and $\|\mathbf{x}-\mathbf{u}\|<\delta$. Then $\left\|x_{i}-u_{i}\right\|<\delta$, and so

$$
\left|\left\|x_{i}\right\|-\left\|u_{i}\right\|-\left\langle\mathbf{x}-\mathbf{u},\left(0, \ldots, \frac{x_{i}}{\left\|x_{i}\right\|}, 0, \ldots, 0\right)\right\rangle\right| \leq \varepsilon\left\|x_{i}-u_{i}\right\| \leq \varepsilon\|\mathbf{x}-\mathbf{u}\| .
$$

This is what we want.

Recall that

- the strong-operator topology on $\mathcal{B}(H)$ is the locally convex one induced by the seminorms $\|\cdot\|_{F}$, where $F=\left\{x_{1}, \ldots, x_{n}\right\}$ is a finitely enumerable subset of $H$ and

$$
\|T\|_{F}=\left(\sum_{i=1}^{n}\left\|T x_{i}\right\|^{2}\right)^{1 / 2}
$$

- the weak-operator topology on $\mathcal{B}(H)$ is the locally convex one induced by the seminorms

$$
T \rightsquigarrow \sum_{i=1}^{n}\left|\left\langle T x_{i}, y_{i}\right\rangle\right|
$$

where $x_{1}, \ldots, x_{n}, y_{1}, \ldots, y_{n}$ are elements of $H$.

Let $H_{n}$ be the direct sum $\bigoplus_{i=1}^{n} H$ of $n$ copies of $H$. To each $T \in \mathcal{B}(H)$ there corresponds an element $\widetilde{T}$ of $\mathcal{B}\left(H_{n}\right)$ defined by

$$
\widetilde{T} \mathbf{x}=\left(T x_{1}, \ldots, T x_{n}\right)
$$

for each $\mathbf{x}=\left(x_{1}, \ldots, x_{n}\right) \in H_{n}$. The mapping $T \rightsquigarrow \widetilde{T}$ is uniformly continuous on $\mathcal{B}(H)$ relative to the weak-operator topologies on $\mathcal{B}(H)$ and $\mathcal{B}\left(H_{n}\right)$ : this readily follows from the identity

$$
\langle\widetilde{T} \mathbf{x}, \mathbf{y}\rangle=\sum_{i=1}^{n}\left\langle T x_{i}, y_{i}\right\rangle .
$$

For each subset $\mathcal{A}$ of $\mathcal{B}(H)$, define

$$
\widetilde{\mathcal{A}}=\{\widetilde{T}: T \in \mathcal{A}\} .
$$

Note that if $\mathcal{A}$ has any of the properties "bounded", "convex", or "weak-operator totally bounded", then $\widetilde{\mathcal{A}}$ has the same property. For example, if $\mathcal{A}$ is weak-operator totally bounded, then the uniform continuity of the mapping $T \rightsquigarrow \widetilde{T}$ relative to the weak-operator topologies ensures that $\widetilde{\mathcal{A}}$ is weak-operator totally bounded ([6], Proposition 2.1.7). Incidentally, the unit ball $\mathcal{B}_{1}(H)$ of $\mathcal{B}(H)$ is weak-operator totally bounded 3 .

Similarly, we define $H_{\infty}$ to be the direct sum $\bigoplus_{i=1}^{\infty} H$ of a sequence of copies of $H$, and we define $\widetilde{T}$ (for each $T \in \mathcal{B}(H)$ ) and $\widetilde{\mathcal{A}}$ in the obvious way. In this case 
also, the properties "bounded", "convex", and "weak-operator totally bounded" transfer from $\mathcal{A}$ to $\widetilde{\mathcal{A}}$.

Lemma 3.7. Let $H$ be a Hilbert space, and let $\mathcal{A}$ be a nonempty subset of $\mathcal{B}(H)$ that is (uniformly) bounded, weak-operator totally bounded, and convex. Let $x_{1}, \ldots, x_{n}$ be elements of $H$, and let $S$ be an element of $\mathcal{B}(H)$. Then the infimum

$$
\inf \left\{\sum_{i=1}^{n}\left\|S x_{i}-T x_{i}\right\|: T \in \mathcal{A}\right\}
$$

exists.

Proof. To begin with, suppose that $\rho\left(S x_{i}, \mathcal{A} x_{i}\right)>0$ for each $i$. (Note that $\mathcal{A} x_{i}$ is located, by Corollary 3.5.) Using the foregoing construction of $H_{n}, \widetilde{T}$, and $\widetilde{\mathcal{A}}$, we see that $\widetilde{\mathcal{A}}$ is bounded, convex, and weak-operator totally bounded. Let $\mathbf{x}=$ $\left(x_{1}, \ldots, x_{n}\right) \in H_{n}$, and let

$$
C=\left\{\left((S-T) x_{1}, \ldots,(S-T) x_{n}\right): T \in \mathcal{A}\right\}=\{(\widetilde{S}-\widetilde{T}) \mathbf{x}: T \in \mathcal{A}\} .
$$

Then $C$ is a bounded, weakly totally bounded, convex subset of $H_{n}$. Define $f$ : $C \rightarrow \mathbf{R}^{0+}$ by

$$
f((\widetilde{S}-\widetilde{T}) \mathbf{x})=\sum_{i=1}^{n}\left\|S x_{i}-T x_{i}\right\| .
$$

Then $f$ is convex and, in view of Lemma 3.6 and the extra supposition at the start of this proof, uniformly differentiable on $C$. So, by Theorem 2.2 , $\inf _{\xi \in C} f(\xi)$ exists. But this infimum is precisely the quantity that we want.

To remove our extra supposition, let $H^{\prime}=H \oplus H$. Given $\delta>0$, define $\mathcal{A}^{\prime}=$ $\mathcal{A} \oplus\left\{\delta^{1 / 2} I\right\}, S^{\prime}=(S, 0)$, and $x_{i}^{\prime}=\left(x_{i}, e\right)(1 \leq i \leq n)$. For each $T \in \mathcal{A}$ we have

$$
\left\|S^{\prime} x_{i}^{\prime}-\left(T, \delta^{1 / 2} I\right) x_{i}^{\prime}\right\|=\left(\left\|S x_{i}-T x_{i}\right\|^{2}+\delta\right)^{1 / 2} \geq \delta^{1 / 2}
$$

so $\rho\left(S^{\prime} x_{i}^{\prime}, \mathcal{A}^{\prime} x_{i}^{\prime}\right)>0$. It follows from the first part of the proof that the quantity

$$
\begin{aligned}
m_{\delta} & =\inf \left\{\sum_{i=1}^{n}\left\|S^{\prime} x_{i}^{\prime}-\left(T, \delta^{1 / 2} I\right) x_{i}^{\prime}\right\|: T \in \mathcal{A}\right\} \\
& =\inf \left\{\sum_{i=1}^{n}\left(\left\|S x_{i}-T x_{i}\right\|^{2}+\delta\right)^{1 / 2}: T \in \mathcal{A}\right\}
\end{aligned}
$$

exists. The desired conclusion now follows from Lemma 3.2

Let $X$ be a vector space over $\mathbf{C}$, and $\left(\|\cdot\|_{i}\right)_{i \in I}$ a family of seminorms defining a locally convex structure on $X$. We say that a subset $S$ of $X$ is located if for each finitely enumerable subset $F$ of $I$ and for each $x \in X$ the infimum

$$
\inf \left\{\sum_{i \in F}\|x-s\|_{i}: s \in S\right\}
$$

exists. In other words, $S$ is located in $X$ if and only if, for each finitely enumerable subset $F$ of $I$, it is located with respect to the pseudometric derived from the seminorm $\sum_{i \in F}\|\cdot\|$. 
Theorem 3.8. Let $H$ be a Hilbert space, and let $\mathcal{A}$ be a nonempty subset of $\mathcal{B}(H)$ that is (uniformly) bounded, weak-operator totally bounded, and convex. Then $\mathcal{A}$ is strong-operator located in $\mathcal{B}(H)$.

Proof. Let $H_{\infty}=\bigoplus_{i=1}^{\infty} H$ be the Hilbert space direct sum of a sequence of copies of $H$, and denote the inner product and norm on $H_{\infty}$ by $\langle\cdot, \cdot\rangle_{\infty}$ and $\|\cdot\|_{\infty}$ respectively. For $1 \leq k \leq m$, let

$$
F_{k}=\left\{x_{1}^{k}, \ldots, x_{n_{k}}^{k}\right\}
$$

be a finitely enumerable subset of $H$, and let

$$
\xi_{k}=\left(x_{1}^{k}, \ldots, x_{n_{k}}^{k}, 0,0,0, \ldots\right) \in H_{\infty} .
$$

For each $S \in \mathcal{B}(H)$ define $\widetilde{S} \in \mathcal{B}\left(H_{\infty}\right)$, and also define $\widetilde{\mathcal{A}}$, as indicated immediately before the statement of Lemma 3.7. Then $\widetilde{\mathcal{A}}$ is (uniformly) bounded, weak-operator totally bounded, and convex in $\mathcal{B}\left(H_{\infty}\right)$. Consider any $S \in \mathcal{B}(H)$. Applying Lemma 3.7 in $H_{\infty}$, we see that

$$
\inf \left\{\sum_{k=1}^{n}\left\|\widetilde{S} \xi_{k}-\widetilde{T} \xi_{k}\right\|: T \in \mathcal{A}\right\}
$$

exists. But this number equals

$$
\inf _{T \in \mathcal{A}} \sum_{k=1}^{n}\left(\sum_{i=1}^{n_{k}}\left\|S x_{i}^{k}-T x_{i}^{k}\right\|^{2}\right)^{1 / 2}=\inf _{T \in \mathcal{A}} \sum_{k=1}^{n}\|S-T\|_{F_{k}} .
$$

This is what we want.

In the classical theory of algebras of operators on a Hilbert space $H$, any subset of $\mathcal{B}_{1}(H)$ is weak-operator totally bounded. Since this is not the case constructively, it seems sensible in the constructive theory of operators to require of a subspace, or subalgebra, $\mathcal{A}$ of $\mathcal{B}(H)$ that its unit ball $\mathcal{A}_{1}$ be weak-operator totally bounded. In that case, although in the infinite-dimensional case it is false that $\mathcal{A}_{1}$ is strong-operator compact, Theorem 3.8 shows that $\mathcal{A}_{1}$ has the potentially useful (if classically vacuous) property of strong-operator locatedness.

\section{REFERENCES}

[1] Errett Bishop, Foundations of Constructive Analysis, McGraw-Hill, New York, 1967. MR 36:4930

[2] Errett Bishop and Douglas Bridges, Constructive Analysis, Grundlehren der Mathematischen Wissenschaften 279, Springer-Verlag, Berlin, 1985. MR 87d:03172

[3] Douglas Bridges and Luminiţa Dediu (Vîţă), Weak-operator continuity and the existence of adjoints, Math. Logic Quarterly 45, no. 2 (1999), 203-206. MR 2000c:46140

[4] Douglas Bridges and Luminița Vîță, Constructing extensions of ultraweakly continuous linear functionals, J. Functional Analysis 178, no. 2 (2000), 421-434. MR 2001k:47101

[5] Douglas Bridges and Fred Richman, Varieties of Constructive Mathematics, London Math. Soc. Lecture Notes 97, Cambridge University Press, 1987. MR 88k:03127

[6] Luminiţa Dediu (Vîţă), The Constructive Theory of Operator Algebras, Ph.D. thesis, University of Canterbury, Christchurch, New Zealand, 2000.

[7] Hajime Ishihara, Constructive compact linear mappings, Bull. London Math. Soc. 21 (1989), 735-740. MR 90k:46171

[8] Hajime Ishihara, Locating subsets of a Hilbert space, Proc. Amer. Math. Soc. 129, no. 5 (2001), 1385-1390. MR 2001h:46132 
[9] Hajime Ishihara and Luminiţa Vîţă, Locating subsets of a normed space, Proc. Amer. Math. Soc. 131, no. 10 (2003), 3231-3239.

[10] A. S. Troelstra and D. van Dalen, Constructivism in Mathematics (Vol. I), North-Holland Publ. Co., Amsterdam, 1988. MR 90e:03002a

Department of Mathematics and Statistics, Private Bag 4800, University of CanterBury, Christchurch, New Zealand

E-mail address: d.bridges@math.canterbury.ac.nz

School of Information Science, Japan Advanced Institute of Science and Technology, TATSUNOKUChI, IshikaWA 923-1292, JAPAN

E-mail address: ishihara@jaist.ac.jp

Department of Mathematics and Statistics, Private Bag 4800, University of CanterBury, Christchurch, New Zealand

E-mail address: Luminita@math.net 